\title{
Effect of Adrenocorticotropic Hormone Stimulation During Simultaneous Bilateral Adrenal Vein Sampling in Primary Aldosteronism
}

Authors

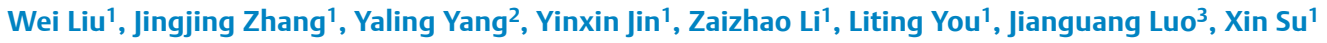

Affiliations

1 National Clinical Research Center for Metabolic Diseases, Institute of Metabolism and Endocrinology, and Department of Metabolism and Endocrinology, The Second Xiangya Hospital of Central South University, Hunan, China

2 Department of Metabolism and Endocrinology, Xuhui Central Hospital, Fudan University, Shanghai, China

3 Department of Radiology, The Second Xiangya Hospital, Central South University, Changsha, Hunan, China

Key words primary aldosteronism, adrenal vein sampling, ACTH stimulation, selectivity index, lateralization index

received 17.12 .2020

accepted 29.04.2021

Bibliography

Horm Metab Res 2021; 53: 364-370

DOI 10.1055/a-1498-6958

ISSN 0018-5043

(c) 2021. Thieme. All rights reserved.

Georg Thieme Verlag KG, Rüdigerstraße 14,

70469 Stuttgart, Germany

Correspondence

Xin Su

National Clinical Research Center for Metabolic Diseases Institute of Metabolism and Endocrinology Department of Metabolism and Endocrinology The Second Xiangya Hospital of Central South University 139 Middle Renmin Road

Changsha

410011

Hunan

China

Tel.:+ 86 13808457300, Fax: + 8613808457300

suxin71@csu.edu.cn
Jianguang Luo

Department of Radiology

The Second Xiangya Hospital

Central South University

Changsha

Hunan

China

1527248158@qq.com

Supplementary Material is available under http://doi.org/10.1055/a-1498-6958.

\section{ABSTRACT}

The aim of the study was to investigate the significance and influence of adrenocorticotropic hormone (ACTH) stimulation in primary aldosteronism (PA) patients with simultaneous bilateral adrenal vein sampling (AVS). All patients diagnosed with PA underwent simultaneous bilateral AVS with ACTH. In 95 patients, the post-ACTH SI significantly increased $(p<0.001)$, and it gradually decreased from $\mathrm{t} 10-\mathrm{t} 30$ after ACTH stimulation $(p<0.001)$. The unsuccessful catheterization decreased after ACTH stimulation. Time points within 20 min after ACTH stimulation were better for sampling, and the selectivity did not increase over longer periods. According to lateralization before and after ACTH stimulation, the patients could be divided into 3 groups ( $U$, unilateral; $B$, bilateral): $U / U, U / B$ or $B / U$, and $B / B$. Compared with the $U / U$ group, in the $U / B$ or $B / U$ and $B / B$ groups, the lateralization index (LI) was lower both at baseline and after ACTH stimulation $(p<0.0001)$, the contralateral index (CLI) was higher after ACTH stimulation $(p<0.003)$, the serum potassium level was higher $(p<0.001)$, and the carbon dioxide combining power ( $\mathrm{CO} 2 \mathrm{CP}$ ) and base excess (BE) levels were lower. In conclusion, in simultaneous bilateral AVS, ACTH stimulation had significant effects on increasing the catheterization selectivity. Lateralization change was observed after stimulation. After ACTH stimulation, fewer patients could be diagnosed with lateralized PA. Patients with consistent lateralized PA showed a more serious phenotype. 


\section{Introduction}

Primary aldosteronism(PA) is the most common cause of secondary hypertension [1]. Patients with PA have higher cardiovascular morbidity and mortality rates than those with essential hypertension [2]. PA is broadly subtyped into bilateral (B) PA (BPA) and unilateral (U) PA (UPA). Unilateral adrenalectomy is the curative treatment for aldosterone-producing adenoma (APA) and provides longterm benefits to patients with UPA [3], including in terms of cardiovascular outcomes [4-6], quality of life [5], and all-cause mortality [7]. Adrenal vein sampling (AVS) is the gold standard test for distinguishing UPA from BPA [8, 9]. However, the best AVS protocol and the interpretation of AVS data still controversial $[10,11]$. According to the recommendations of the Adrenal Vein Sampling International Study (AVIS), clinical centers can choose simultaneous or non-simultaneous AVS based on the level of interventional medicine, and in non-simultaneous AVS, the use of adrenal glandular cortex excitement [synthetic adrenocorticotropic hormone (ACTH ) stimulation] is recommended [10]. However, the impact of ACTH on PA lateralization is controversial. As one of the few clinical centers in China that can successfully complete simultaneous bilateral AVS, the Second Xiangya Hospital of Central South University has been using simultaneous bilateral AVS with blood collection before and after ACTH stimulation for this determination. The purpose of this study was to explore the role of simultaneous bilateral AVS with ACTH stimulation in the diagnosis of PA and related optimization schemes.

\section{Patients and Methods}

\section{Patients}

We prospectively studied all patients with confirmed PA who underwent AVS at the Second Xiangya Hospital of Central South University (Changsha, Hunan, China) between November 2017 and November 2019. A total of 95 consecutive patients were enrolled. We recorded the clinical data of all patients. Patients provided written informed consent for AVS, and data analysis was approved by the Xiangya Second Hospital Clinical Ethics Committee (2017sk2022).

\section{Clinical assessment}

The diagnosis of PA was made according to the diagnostic criteria of the Endocrine Society guidelines [8]. Before screening, all patients were evaluated after discontinuing all antihypertensive drugs, except for non-dihydropyridine calcium channel blockers and alpha-adrenergic blockers, such as spironolactone, eplerenone (mineralocorticoid receptor antagonist) and potassium (K) diuretics for at least 4 weeks, and beta blockers, angiotensin-converting enzyme inhibitors and angiotensin receptor antagonists for at least 2 weeks. Hypokalemia was corrected before AVS.

Renin-angiotensin-aldosterone system (RAAS) system screening included measurement of the plasma aldosterone concentration (PAC) and plasma renin activity (PRA) and calculation of the aldosterone-renin ratio (ARR). Patients with a $P A C \geq 10 \mathrm{ng} / \mathrm{dl}$ and an $A R R \geq 20 \mathrm{ng} / \mathrm{dl} / \mathrm{ng} / \mathrm{ml} / \mathrm{h}$ underwent a further captopril challenge test (CCT) and/or saline infusion test.
A low-dose dexamethasone suppression test was performed to exclude Cushing's syndrome.

All assays were performed in the biochemistry laboratory at the Second Xiangya Hospital of Central South University. The PAC was measured using a chemiluminescence assay (Maglumi 2000 Plus, China); the intra-assay and inter-assay CV for the PAC was $\leq 5 \%$ and $\leq 10 \%$, respectively. The PRA was also measured by chemiluminescence assay (Maglumi 2000 Plus, China); the intra-assay and inter-assay CV for the PAC was $\leq 15 \%$ and $\leq 10 \%$, respectively. The serum cortisol level was measured by a competitive chemiluminescent immunoassay (Maglumi 2000 Plus, China), with a CV of 5.1$7.1 \%$. Computed tomography (CT) scans of the adrenal glands with contiguous $2.5 \mathrm{~mm}$ slices were performed in all patients with PA before undergoing AVS.

AVS was performed by one experienced interventional radiologist at the Second Xiangya Hospital of Central South University. Samples were obtained both from the inferior vena cava (IVC) and bilateral adrenal veins (AVs) before $(t-5, t 0)$ and 10,20 , and 30 min after (t10, t20, and t30) ACTH stimulation. ACTH was injected as a $0.125 \mathrm{mg}$ bolus followed by continuous infusion $(0.125 \mathrm{mg} / \mathrm{h})$. The success of catheterization was determined by the selectivity index (SI, derived from AV/IVC cortisol concentrations) $\geq 2$ before and $\geq 3$ after ACTH stimulation. The source of aldosterone excess was assessed by the lateralization index (LI), which was calculated by dividing the dominant adrenal cortisol-corrected aldosterone level $(\mathrm{A} / \mathrm{C})_{\text {DOM }}$ by that of the opposite side $(\mathrm{A} / \mathrm{C})_{\text {OPp. }}$ UPA was diagnosed if the LI was $\geq 2$ before and $\geq 4$ after ACTH administration. The contralateral index (CLI) was defined as (aldosterone/cortisol) non-domi- $_{\text {- }}$ nant AV/(aldosterone/cortisol) IVc. For analysis, PRA values $<0.2 \mathrm{ng} /$ $\mathrm{ml}$ per hour were set to 0.2 .

\section{Statistical analysis}

Statistical Package for the Social Sciences, version 23.0, for Windows (SPSS) and GraphPad Prism 5.00 (GraphPad Prism Software, Inc.) were used for plotting the results. Descriptive statistics are reported as the mean \pm SD or median [interquartile range (IQR)], as appropriate. Classification variables are expressed as numbers and percentages. A normality test was used to determine the distribution of the variables. ANOVA was used to compare normally distributed variables among groups. Kruskal-Wallis test was used to compare variables with a non-normal distribution among groups. For categorical variables, we used the Fisher exact test. Wilcoxon signed-rank test was used to compare preoperative and postoperative values. All tests were two tailed; $p$-values $<0.05$ were considered statistically significant.

\section{Results}

\section{Clinical characteristics of participants}

The clinical characteristics of all patients are presented in $\triangleright$ Table 1. Between November 2017 and November 2019, 104 patients diagnosed with PA underwent AVS at our institution. 9 cases with incomplete blood sampling data were excluded, 95 were ultimately included in the present study. The median age of the study participants was 49 (range, $21-70$ ) years, and 49 (52\%) were men. The median duration of hypertension was 7.5 (IQR: 2.8, 10.0) years, 
and the mean blood pressure (BP) was $158( \pm 19) / 96( \pm 15) \mathrm{mmHg}$. The mean serum $\mathrm{K}$ level was $3.10 \pm 0.44 \mathrm{mmol} / \mathrm{l}$, with 79 (83\%) patients having a serum $\mathrm{K}$ level $<3.5 \mathrm{mmol} / \mathrm{l}$. The median ARR was 320.5 (IQR: 110.9, 858.8), and 64-detector row CT scans were performed in all patients. A sum of 74 (78\%) patients had unilateral lesions, 19 (20\%) had bilateral adrenal nodules, and 2 (2\%) had normal-appearing adrenals on CT. The median of the longest diameter of the lesion on CT was 15 (IQR: 11, 20) mm ( Table 1).

- Table 1 Clinical characteristics of patients.

\begin{tabular}{|l|l|}
\hline Variables & \\
\hline Age, median (range), years & $49(21,70)$ \\
\hline Male sex, no. (\%) & $49(52 \%)$ \\
\hline $\begin{array}{l}\text { Duration of hypertension, median (IQR), } \\
\text { years }\end{array}$ & $7.5(2.8-10.0)$ \\
\hline Systolic BP, mmHg & $158 \pm 19$ \\
\hline Diastolic BP, mmHg & $96 \pm 15$ \\
\hline Serum K+, mmol/l & $3.10 \pm 0.44$ \\
\hline Serum $\mathrm{K}^{+<3.50 ~ m m o l / I, ~ n ~(\%) ~}$ & $79(83 \%)$ \\
\hline ARR, median (IQR) & $320.5(110.9,858.8)$ \\
\hline CT results, no. (\%) & $74(78 \%)$ \\
\hline Unilateral lesion & $19(20 \%)$ \\
\hline Bilateral lesion & $2(2 \%)$ \\
\hline Negative & $15(11,20)$ \\
\hline Lesion diameter on CT, median (IQR), mm &
\end{tabular}

Data are presented as the mean \pm SD or median (IQR), as appropriate. BP: Blood pressure; ARR: Aldosterone-renin ratio.

\section{Analysis of baseline vs post-ACTH AVS hormone ratios}

Comparison of selectivity index (SI) before and after ACTH administration

ACTH significantly stimulated cortisol (12.0- to 14.9-fold on the right, 10.0- to 13.2-fold on the left) and aldosterone (4.0- to 7.0fold on the right, 4.3- to 4.6-fold on the left), as measured in the AVs, the levels of which were both higher than those in the IVC (1.6to 2.2-fold for cortisol, 1.2- to 1.5-fold for aldosterone) (Supplementary Table $1 \mathrm{~S}$ ). The median SI significantly increased on the left side from 2.95 (IQR: 1.88-12.89) at baseline [ $-5 \mathrm{~min},(\mathrm{t}-5)$ ], and 3.62 (IQR: 1.89-11.04) at 0 minute (t0) to 46.31 (IQR: $15.05-$ $70.39)$ at 10 minutes (t10), 32.34 (IQR: $14.90-51.70)$ at 20 minutes (t20) and 29.79 (IQR: 12.09-44.40) at 30 minutes (t30) postACTH; on the right side, the median SI was 4.24 (IQR: 2.20-12.02) at t-5, 3.85 (IQR: 2.31-11.65) at t0, 60.19 (IQR: 14.55-89.19) at t10, 45.69 (IQR: 17.31-77.69) at t20, and 41.85 (IQR: 17.22$64.06)$ at $\mathrm{t} 30$ ( $\mathrm{p}<0.001$ post-ACTH vs baseline). There was no significant difference in the SI between $\mathrm{t}-5$ and $\mathrm{t} 0$, and the SI gradually decreased from $\mathrm{t} 10$ to $\mathrm{t} 20$ and $\mathrm{t} 30$ after stimulation with $\mathrm{ACTH}$ $(p<0.001)$ ( $>$ Fig. 1).

According to the standard of successful catheterization, the unsuccessful catheterization rate was $20.0 \%$ (19/95) and $5.3 \%$ (5/95) on the left and $12.6 \%$ (12/95) and 6.3\% (6/95) on the right at baseline and after ACTH stimulation, respectively. In all cases, the rate of unsuccessful catheterization on either left or right side at baseline was $28.4 \%$ (27/95) and $11.6 \%$ (11/95) after ACTH stimulation. ACTH stimulation effectively reduced the rate of unsuccessful catheterization for AVS ( $\triangleright$ Fig. 2).

Unsuccessful catheterization after ACTH stimulation was observed in $6.3 \%, 10.5 \%$, and $10.5 \%$ of patients at $\mathrm{t} 10$, $\mathrm{t} 20$, and $\mathrm{t} 30$, respectively, on the left side and in $11.6 \%, 11.6 \%$, and $16.8 \%$ at t 10 , $\mathrm{t} 20$, and $\mathrm{t} 30$ on the right side, respectively. The lowest and highest rate of unsuccessful catheterization was observed at $\mathrm{t} 10$ and $\mathrm{t} 30$,

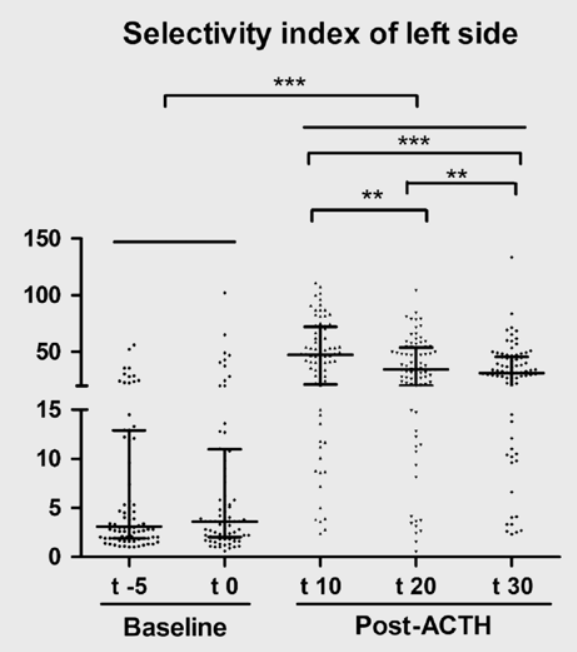

\section{Selectivity index of right side}

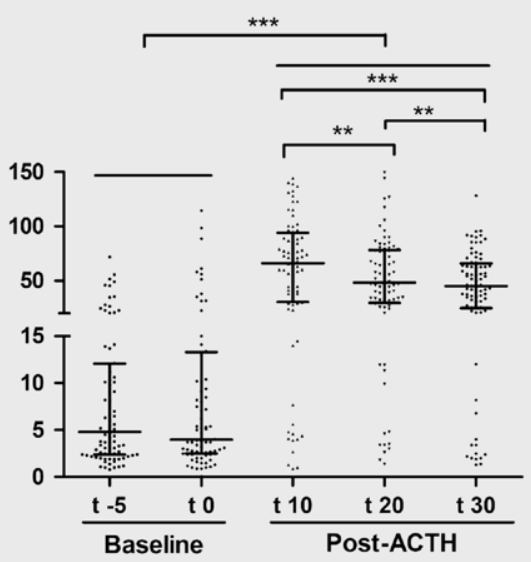

Fig. 1 SI of the left and right adrenals. ${ }^{*} \mathrm{p}<0.05 ;{ }^{*}{ }^{*} \mathrm{p}<0.01 ;{ }^{* * *} \mathrm{p}<0.001$. 
respectively. There was no difference in catheterization selectivity between $\mathrm{t} 10+\mathrm{t} 20$ and $\mathrm{t} 10+\mathrm{t} 20+\mathrm{t} 30$. The results show no increase in selectivity 30 minutes after ACTH stimulation (• Fig. $\mathbf{3}$ ).

\section{Influence of ACTH stimulation on LI}

\section{Lateralization at baseline and after ACTH stimulation}

A total of 11 patients with unsuccessful catheterization (unilateral $n=5$, bilateral $n=6$ ) both before and after ACTH stimulation were excluded. Of the remaining 84 patients, 63 patients underwent selective catheterization both before and after ACTH stimulation. According to lateralization, these patients could be divided into 3 groups (U, unilateral; $B$, bilateral): $U / U(n=35,55.6 \%) ; U / B$ or $B / U$ ( $[n=20,31.7 \%]$, including $U / B(n=18), B / U(n=2)]$; and $B / B(n=8$, $12.7 \%)$. Compared with the $U / U$ group, in the $U / B$ or $B / U$ and $B / B$ groups, the LI was lower both at baseline and after ACTH stimulation $(p<0.0001)$, the contralateral index $(C L I)$ was higher after ACTH stimulation $(p<0.003)$, the serum potassium level was higher $(\mathrm{p}<0.001)$, and the carbon dioxide combining power (CO2CP) and base excess (BE) levels were lower ( $\triangleright$ Table 2 ). There were no differences in BP, age, sex, or aldosterone or cortisol concentration among the groups (data not shown). Patients in $\mathrm{U} / \mathrm{B}$ and $\mathrm{B} / \mathrm{U}$ groups did not choose surgery.

\section{Comparison of AVS and CT results}

Among the 43 patients with the same lateral conditions before and after ACTH stimulation, unilateral PA were found in $81.4 \%$ (35/43),

Rate of unsuccessful catheterization

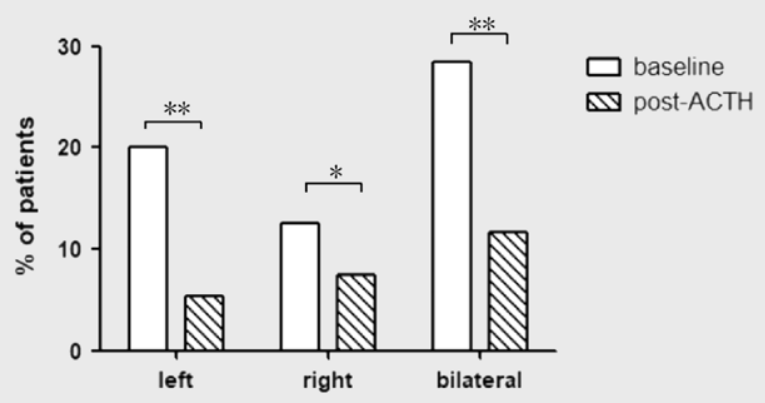

- Fig. 2 Proportion of patients with unsuccessful catheterization for AVS. ${ }^{*} \mathrm{p}<0.05 ;{ }^{*}{ }^{*} \mathrm{p}<0.01$. bilateral PA were found in $18.6 \%$ (8/43), and the CT coincidence rate was $51.2 \%$ (22/43) (Supplementary Table 2S).

\section{Postoperative follow-up}

Among the 35 patients with lateralized hyperaldosteronism both before and after ACTH stimulation, 28 underwent surgery; among these patients, the CT and AVS results were different in 8 (all 8 were bilateral on CT; on AVS, 6 lateralized hyperaldosteronism were on the right and 2 were on the left). Twenty-two patients (78.6\%) had a normal blood potassium level on the second day after the operation. The potassium and blood pressure levels were significantly improved $(p<0.001)$ ( $\triangleright$ Table 3$)$.

\section{Discussion}

PA is the most common cause of secondary hypertension, and UPA can be cured with surgery; thus, differentiating lateralized from bilateral PA is important. AVS has high sensitivity (95\%) and specificity (100\%) for detecting lateralized aldosterone excess, and is now the gold standard for the lateral diagnosis of PA [8]. However, the process of performing AVS and interpreting the results have yet to be standardized [11]. Some centers use unilateral sequential sampling, while others use simultaneous bilateral sampling [12]. Different centers perform AVS with or without ACTH stimulation $[13,14]$. There are no specific guidelines or recommendations regarding whether ACTH stimulation is necessary $[15,16]$. One of the important reasons for the uncertain efficacy of surgery for PA is the inconsistency among AVS procedures and the interpretation of AVS results [17]. Additionally, surgical outcomes in patients with lateralized PA are closely related to the procedure and diagnostic criteria [18]. Therefore, it is of great clinical value to establish standards for performing AVS procedures and analyzing AVS results.

In our study, bilateral simultaneous AVS with ACTH stimulation was performed by one experienced radiologist, and laboratory tests were performed by the same methods. Our center is one of the few institutions to collect samples at 5 time points before and after ACTH stimulation. The same standard sampling procedure was applied in every patient. Therefore, we can evaluate differences in results between different conditions in the same patient and evaluate the role of ACTH in simultaneous bilateral AVS.

In AVS, the SI is used to determine whether a sufficient cortisol gradient is obtained between the adrenal gland and the periphery
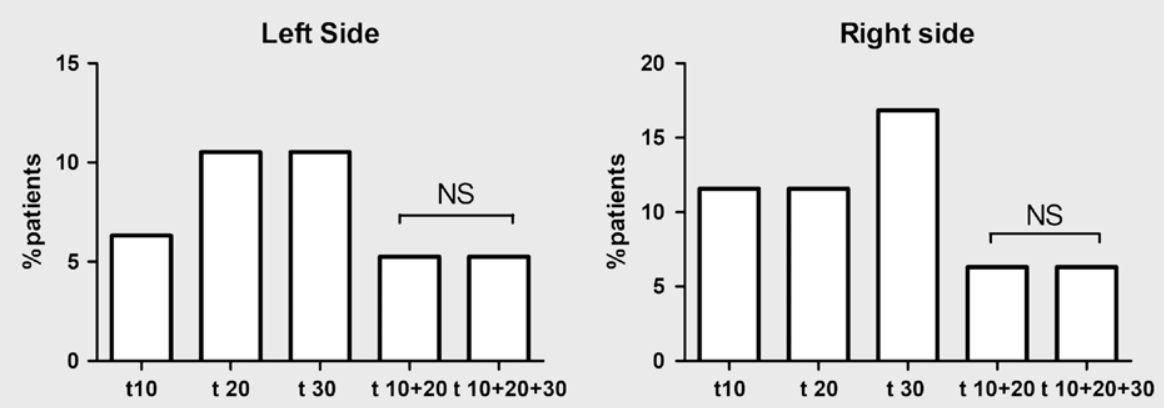

Fig. 3 Proportion of patients with unsuccessful catheterization for AVS at each sampling time point. NS: Non-significant difference. 
- Table 2 Characteristics of study participants by laterality before and after ACTH stimulation.

\begin{tabular}{|c|c|c|c|c|}
\hline & $\mathrm{U} / \mathrm{U}$ & $\mathrm{U} / \mathrm{B}$ or $\mathrm{B} / \mathrm{U}$ & B/B & p-value ${ }^{a}$ \\
\hline No. of participants (\%) & $35(55.6)$ & $20(31.7)$ & $8(12.7)$ & \\
\hline Age, median (range), years & $48(33-65)$ & $47.5(30-71)$ & $50(28-68)$ & 0.945 \\
\hline Male sex, no. (\%) & $18(51)$ & $9(45)$ & $3(38)$ & 0.858 \\
\hline \multicolumn{5}{|l|}{ LI, median, (IQR) } \\
\hline Baseline & $14.02(7.12-35.73)$ & $3.57^{* * *}(2.68-9.19)$ & $1.25^{* * *}(1.20-1.76)$ & \\
\hline Post-ACTH & $15.07(7.12-47.63)$ & $1.72^{* * *}(1.24-2.26)$ & $1.18^{* * *}(1.04-1.77)$ & \\
\hline \multicolumn{5}{|l|}{ CLI, median, (IQR) } \\
\hline Baseline & $0.28(0.09-0.50)$ & $0.41(0.23-1.07)$ & $0.56(0.26-1.32)$ & 0.29 \\
\hline Post-ACTH & $0.10(0.06-0.23)$ & $0.47^{* *}(0.15-0.82)$ & $0.37^{* *}(0.15-1.73)$ & \\
\hline Serum potassium, median, (IQR) & $2.87(2.63-3.13)$ & $3.35^{* * *}(2.99-3.58)$ & $3.60^{* * *}(2.98-3.69)$ & \\
\hline $\mathrm{BE}$ & $4.3(2.0-6.7)$ & $1.45(0.75-3.0)$ & $-0.4^{* *}(-0.9$ to -0.5$)$ & \\
\hline $\mathrm{CO} 2 \mathrm{CP}$ & $26.6(24.4-29.8)$ & $24.3^{*}(21.3-26.3)$ & $26.3(24.2-28.7)$ & \\
\hline
\end{tabular}

- Table 3 Blood pressure and potassium levels of patients the day after the operation.

\begin{tabular}{|c|c|c|c|}
\hline & Preoperative & Postoperative & p-Value \\
\hline $\mathrm{SBP}, \mathrm{mmHg}$ & $163 \pm 20$ & $130 \pm 14$ & $<0.001$ \\
\hline $\mathrm{DBP}, \mathrm{mmHg}$ & $97 \pm 17$ & $79 \pm 11$ & $<0.001$ \\
\hline $\mathrm{K}^{+}, \mathrm{mmol} / \mathrm{l}$ & $2.85 \pm 0.38$ & $3.91 \pm 0.57$ & $<0.001$ \\
\hline
\end{tabular}

Wilcoxon signed-rank test was used to compare preoperative and postoperative values.

and to determine whether the catheter is located near the AVs. Since in about $12 \%$ patients, the right $A V$ shares egress into the IVC with hepatic accessory veins [19], samples collected from the right $\mathrm{AV}$ maybe an admixture of blood with adrenal gland and liver(which have a much lower concentration of cortisol) and imply a low SI. Catheterization of the left $A V$ is easier. However, this vein also receives blood from the phrenic vein. Therefore, anatomical and physiological factors may influence the SI even if the catheterization was correct at baseline; this problem could be solved by stimulating cortisol secretion through ACTH. At present, there are no standardized ACTH stimulation protocols, and methods of ACTH stimulation include a high-dose bolus, infusion after a bolus and a bolus after infusion [13-16, 20-25]. With a very low-dose bolus [16] after infusion and sustained infusion ( $250 \mu \mathrm{g} / 30 \mathrm{~min})$ [20], as performed at some centers, no difference was observed in the SI between baseline and after ACTH. With the other methods of administration, the SI was significantly increased, and the greatest increase in adrenal cortisol secretion was stimulated by infusion after a bolus
[13-16, 20-25]. Our center performs simultaneous bilateral catheterization using a medium-dose ACTH bolus $(125 \mu \mathrm{g})$, followed by a continuous intravenous infusion $(125 \mu \mathrm{g} / \mathrm{h})$; throughout the process, the catheter is not moved. Our results show that the increase in the rate of selective catheterization with ACTH stimulation is not directly related to the technology and is more likely to be related to the physiological and anatomical characteristics of the AVs. It is important to find a way to determine whether the catheter is in the correct position. Our results support that in simultaneous bilateral AVS, the continuous intravenous infusion of ACTH after a mediumdose bolus can effectively reduce the rate of unsuccessful catheterization. At the same time, we found that the highest median SI (approximately 15 -fold the baseline value) appeared at 10 min after ACTH stimulation and then gradually decreased to 20 and $30 \mathrm{mi}-$ nutes after ACTH stimulation, and the SI at $\mathrm{t} 30$ did not increase the selectivity. Therefore, we think the proportion of patients with selective catheterization can be significantly increased by ACTH stimulation and that sampling at 10 and 20 min after a bolus is sufficiently effective.

After catheter placement, the cortisol gradient can be used to correct the ratio of the adrenal and peripheral aldosterone levels $(\mathrm{A} / \mathrm{C})$ for adrenal blood dilution; then, the corrected ratio can be used to compare the two AVs with each other (LI) and/or with the peripheral circulation (CLI) and determine whether the aldosterone secretion is lateralized. The use of ACTH is remains controversial. Some studies have found that ACTH reduces the accuracy of the judgement of lateralization [16, 25], while others have considered ACTH beneficial for making a diagnosis. Mathur et al. [26] found that although lateralization could be determined in the majority of patients with only pre-ACTH stimulation values, lateralization was most accurately determined by AVS after ACTH stimulation. Kline et al. [13] demonstrated that ACTH infusion may help less experienced AVS operators obtain clinically useful results by maximizing 
the recognition of successful sampling. Rossi et al. [25] found that ACTH improved the assessment of selectivity but exerted a confounding effect on the LI; the LI may be inverted. Our study also shows a phenomenon of lateralization change, and interestingly, the groups of patients with different responses to ACTH stimulation seemed to have different degrees of clinical manifestations. Patients showing results consistent with a unilateral lesion showed a more serious phenotype, including a higher $\mathrm{LI}$, a lower $\mathrm{CLI}$, a lower potassium level, and higher $\mathrm{CO} 2 \mathrm{CP}$ and BE levels. In the $\mathrm{U} / \mathrm{B}$ or B/U group, these factors were between those in the $U / U$ and $B / B$ groups. this group represents an intermediate state between lateralized and bilateral PA which is consistent with previous research findings [27, 28]. Wannachalee et al. [27] found the severity of PA and the potential molecular characteristics of APAs seem to influence the effect of ACTH on the $\mathrm{LI}$ in patients with PA. Patients with KCNJ5 mutations tend to have a higher $\mathrm{LI}$ at baseline that decreases after ACTH stimulation. Our study supports the idea that there is an intermediate state regarding the response to ACTH in PA. Pathological analysis and genetic testing should be included in future research. The rate of agreement between CT and AVS in our research was $51.2 \%$ (22/43), which is consistent with that in previous studies. We monitored the blood potassium and blood pressure levels of the patients who underwent surgery according to the AVS results for a short period of time postoperatively; both the blood potassium and BP level improved. However, the long-term efficacy still needs to be observed.

In conclusion, our study shows that in simultaneous bilateral AVS with continuous ACTH infusion after a bolus of ACTH has significant effects on increasing the selectivity of catheterization. Sampling should be performed within 20 minutes after ACTH stimulation, as the selectivity does not increase over longer periods. After stimulation, $31.7 \%$ of patients showed a change in lateralization, with 18 patients ( $28.6 \%$ ) changing from lateralized to bilateral; after ACTH stimulation, fewer patients could be diagnosed with lateralized hyperaldosteronism. The rate of agreement between the CT and AVS results was only $51.2 \%$. In patients who underwent surgery according to the AVS results, the blood potassium and BP levels both improved within a short period of time.

\section{Limitation}

This was a single-center study with a small sample size, and a larger, a multicenter prospective study using standardized procedures is needed to further clarify the clinical effect of ACTH on PA lateralization. Further pathological and genetic evidence is required, as well as long-term follow-up analysis.

\section{Funding}

This study was supported by the Hunan Provincial Science and Technology Department (2017sk2022, 2020JJ4806, 2020JJ5816).

\section{Conflict of Interest}

The authors declare that they have no conflict of interest.

\section{References}

[1] Rossi GP, Bernini G, Caliumi C et al. A prospective study of the prevalence of primary aldosteronism in 1,125 hypertensive patients. J Am Coll Cardiol 2006; 48: 2293-2300

[2] Milliez P, Girerd X, Plouin PF et al. Evidence for an increased rate of cardiovascular events in patients with primary aldosteronism. J Am Coll Cardiol 2005; 45: 1243-1248

[3] Williams TA, Lenders JWM, Mulatero P et al. Outcomes after adrenalectomy for unilateral primary aldosteronism: an international consensus on outcome measures and analysis of remission rates in an international cohort. Lancet Diabetes Endocrinol 2017; 5: 689-699

[4] Catena C, Colussi G, Lapenna R et al. Long-term cardiac effects of adrenalectomy or mineralocorticoid antagonists in patients with primary aldosteronism. Hypertension 2007; 50: 911-918

[5] Ahmed AH, Gordon RD, Sukor N et al. Quality of life in patients with bilateral primary aldosteronism before and during treatment with spironolactone and/or amiloride, including a comparison with our previously published results in those with unilateral disease treated surgically. J Clin Endocrinol Metab 2011; 96: 2904-2911

[6] Hundemer GL, Curhan GC, Yozamp N et al. Incidence of atrial fibrillation and mineralocorticoid receptor activity in patients with medically and surgically treated primary aldosteronism. JAMA Cardiol 2018; 3: 768-774

[7] Reincke M, Fischer E, Gerum S et al. Observational study mortality in treated primary aldosteronism: The German Conn's registry. Hypertension 2012; 60: 618-624

[8] Funder JW, Carey RM, Mantero F et al. The Management of Primary Aldosteronism: Case Detection, Diagnosis, and Treatment: An Endocrine Society Clinical Practice Guideline. J Clin Endocrinol Metab 2016; 101: 1889-1916

[9] Young WF, Stanson AW, Thompson GB et al. Role for adrenal venous sampling in primary aldosteronism. Surgery 2004; 136: 1227-1235

[10] Rossi GP, Barisa M, Allolio B et al. The adrenal vein sampling international study (AVIS) for identifying the major subtypes of primary aldosteronism. J Clin Endocrinol Metab 2012; 97: 1606-1614

[11] Mulatero P, Bertello C, Sukor $\mathrm{N}$ et al. Impact of different diagnostic criteria during adrenal vein sampling on reproducibility of subtype diagnosis in patients with primary aldosteronism. Hypertension 2010; 55: 667-673

[12] Carr CE, Cope C, Cohen DL et al. Comparison of sequential versus simultaneous methods of adrenal venous sampling. J Vasc Interv Radiol 2004; 15: 1245-1250

[13] Kline GA, So B, Dias VC et al. Catheterization during adrenal vein sampling for primary aldosteronism: Failure to use (1-24) ACTH may increase apparent failure rate. J Clin Hypertens 2013; 15: 480-484

[14] Sung TY, Alobuia WM, Tyagi MV et al. Adrenal vein sampling to distinguish between unilateral and bilateral primary hyperaldosteronism: to ACTH stimulate or not? J Clin Med 2020; 9: 1447

[15] Laurent I, Astere M, Zheng $F$ et al. Adrenal venous sampling with or without adrenocorticotropic hormone stimulation: A meta-analysis. J Clin Endocrinol Metab 2018; 104: 1060-1068

[16] Seccia TM, Miotto D, De Toni R et al. Adrenocorticotropic hormone stimulation during adrenal vein sampling for identifying surgically curable subtypes of primary aldosteronism: comparison of 3 different protocols. Hypertension 2009; 53: 761-766

[17] Wang TS, Kline G, Yen TW et al. A Multi-institutional comparison of adrenal venous sampling in patients with primary aldosteronism: Caution advised if successful bilateral adrenal vein sampling is not achieved. World J Surg 2018; 42: 466-472 
[18] Dekkers T, Prejbisz A, Kool LJS et al. Adrenal vein sampling versus CT scan to determine treatment in primary aldosteronism: An outcomebased randomised diagnostic trial. Lancet Diabetes Endocrinol 2016; 4: 739-746

[19] Miotto D, De Toni R, Pitter $G$ et al. Impact of accessory hepatic veins on adrenal vein sampling for identification of surgically curable primary aldosteronism. Hypertension 2009; 54: 885-889

[20] Wolley M], Ahmed AH, Gordon RD et al. Does ACTH improve the diagnostic performance of adrenal vein sampling for subtyping primary aldosteronism? Clin Endocrinol (Oxf) 2016; 85: 703-709

[21] Durivage C, Blanchette R, Soulez $G$ et al. Adrenal venous sampling in primary aldosteronism: multinomial regression modeling to detect aldosterone secretion lateralization when right adrenal sampling is missing. J Hypertens 2017; 35: 362-368

[22] El Ghorayeb N, Mazzuco TL, Bourdeau I et al. Basal and Post-ACTH aldosterone and its ratios are useful during adrenal vein sampling in primary aldosteronism. J Clin Endocrinol Metab 2016; 101: 1826-1835

[23] Sonoyama T, Sone M, Tamura N et al. Role of endogenous ACTH on circadian aldosterone rhythm in patients with primary aldosteronism. Endocr Connect 2014; 3: 173-179
[24] Monticone S, Satoh F, Giacchetti G et al. Effect of adrenocorticotropic hormone stimulation during adrenal vein sampling in primary aldosteronism. Hypertension 2012; 59: 840-846

[25] Hundemer GL, Curhan GC, Yozamp N et al. Incidence of atrial fibrillation and mineralocorticoid receptor activity in patients with medically and surgically treated primary aldosteronism. JAMA Cardiol 2018; 3: 768-774

[26] Mathur A, Kemp CD, Dutta U et al. Consequences of adrenal venous sampling in primary hyperaldosteronism and predictors of unilateral adrenal disease. J Am Coll Surg 2010; 211: 384-390

[27] Wannachalee T, Zhao L, Nanba K et al. Three discrete patterns of primary aldosteronism lateralization in response to cosyntropin during adrenal vein sampling. J Clin Endocrinol Metab 2019; 104: 5867-5876

[28] Turcu AF, Wannachalee T, Tsodikov A et al. Comprehensive analysis of steroid biomarkers for guiding primary aldosteronism subtyping. Hypertension 2020; 75: 183-192 